\title{
Hypertrophic nonunion of the ulna in a child: treatment with an elastic stable intramedullary nail without bone graft
}

\author{
Abdul Halim Abd Rashid · Sharaf Ibrahim
}

Received: 13 November 2009/ Accepted: 29 April 2010/Published online: 19 May 2010

(C) The Author(s) 2010. This article is published with open access at Springerlink.com

\begin{abstract}
Nonunion following diaphyseal forearm fracture is an uncommon complication in children. Compression plate fixation with bone grafting has been the standard method to treat this complication. We report a case of hypertrophic nonunion of the ulna in a child who was treated surgically using an elastic stable intramedullary nail (ESIN) without bone grafting. The nonunion healed 4 months after surgery.
\end{abstract}

Keywords Paediatric diaphyseal fracture - Ulna ·

Nonunion · Elastic stable intramedullary nail · Bone graft

\section{Introduction}

Forearm fractures are common in children. Most of these fractures can be treated nonsurgically with excellent functional outcomes. Complications such as nonunion are uncommon and not well described. We report a case of hypertrophic nonunion of the ulna in a child which was successfully treated with a close elastic stable intramedullary nail (ESIN). To the best of our knowledge, there has been no published report, regarding the use of ESIN in treating hypertrophic nonunion in a child.

A. H. Abd Rashid $(\bowtie) \cdot$ S. Ibrahim

Department of Orthopaedics and Traumatology,

Faculty of Medicine, Universiti Kebangsaan Malaysia,

Jalan Yaacob Latif, 56000 Kuala Lumpur, Malaysia

e-mail: drhalimrashid@yahoo.com

\section{Case report}

A 12-year-old boy was admitted to our hospital after a fall while playing on a see-saw in the playground. He sustained a closed fracture of the left radius and ulna. He was treated with open reduction and intramedullary Kirschner (K) wiring of the radius and ulna (Fig. 1a, b). The left forearm was protected with a posterior plaster slab. The intramedullary $\mathrm{K}$ wires were removed 6 weeks after surgery. The posterior plaster slab was removed 2 weeks later to allow mobilization of the forearm. The radius united within 6 months but the ulna developed a hypertrophic nonunion (Fig. 2a, b). A closed, antegrade ESIN size $2.5 \mathrm{~mm}$ was inserted without bone grafting. We used a $2.5-\mathrm{mm} \mathrm{K}$ wire to create a passage through the hypertrophic nonunion to facilitate the nail insertion. However, there was no formal reaming done. A $2.5-\mathrm{mm}$ ESIN was chosen as it occupies about $80 \%$ of the narrowest diameter of the ulna to provide a stable fixation. The forearm was not immobilized postoperatively. Four months after nailing, the ulna nonunion completely healed (Fig. 3a, b). The ESIN was removed after 1 year. He has full supination and pronation of the affected forearm (Fig. 4a, b).

\section{Discussion}

Fracture nonunion in a child is uncommon [1-3] as fracture healing in children is usually uncomplicated with an excellent remodelling potential [1]. There are several factors that may have led to the nonunion in our patient.

First, the initial open reduction of the ulna with stripping of the periosteum may have devascularized the ulna. The mid-diaphyseal region has been identified as a "watershed area" in the intraosseous blood supply of the ulna $[2,4]$. 

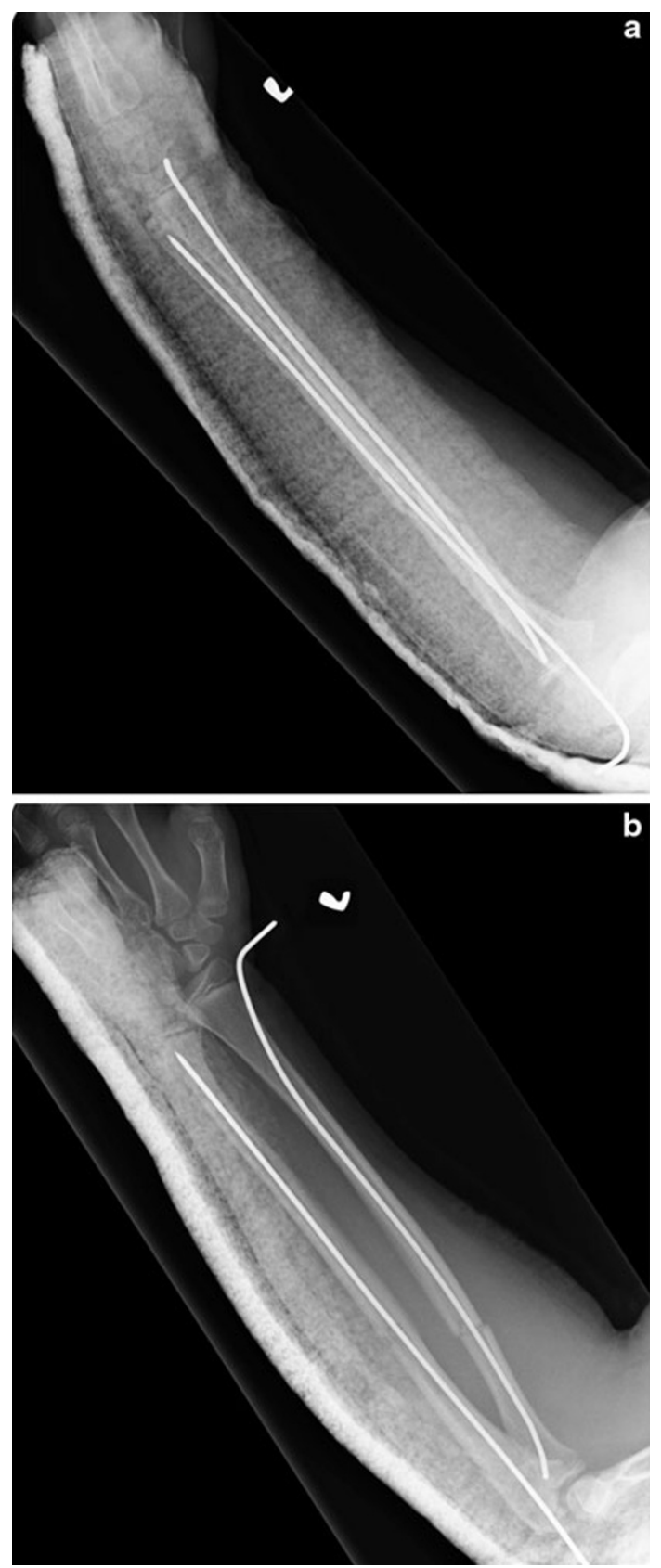

Fig. 1 a Lateral view of the radius and ulna with intramedullary $\mathrm{K}$ wires. b Anteroposterior view of the radius and ulna with intramedullary $\mathrm{K}$ wires

The periosteal blood supply from the anterior and posterior interoseous arteries is the main vascular supply to this region [4]. Giebel et al. [5] have shown that performing open reduction and plate osteosynthesis in the forearm will jeopardize the blood supply to that particular area resulting in delayed union or nonunion.

Second, 8 weeks of immobilization was probably inadequate in a 12-year-old boy resulting in the hypertrophic nonunion of the ulna. This is in contrast to Yung et al. who
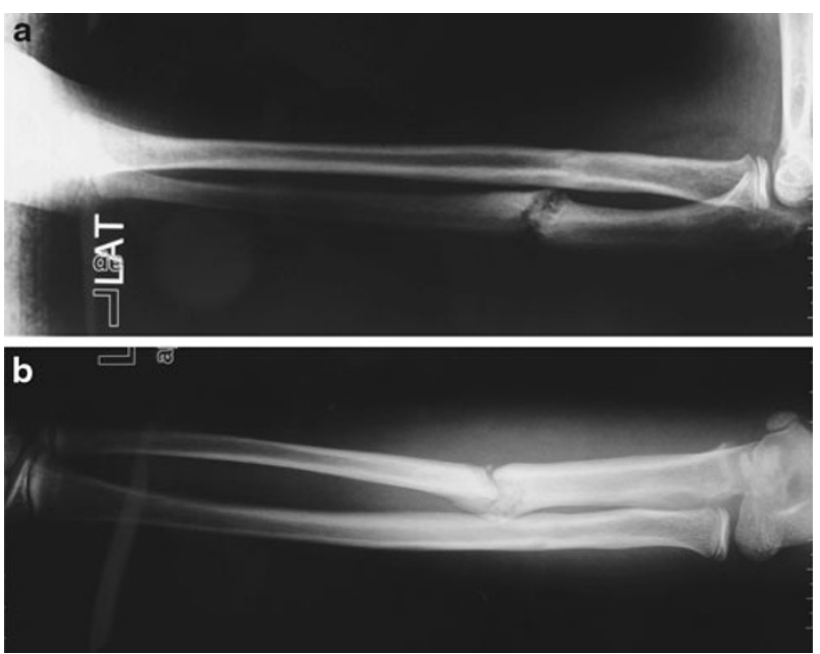

Fig. 2 a Hypertrophic nonunion of left ulna 6 months after injurylateral view. b Hypertrophic nonunion of left ulna 6 months after injury-anteroposterior view
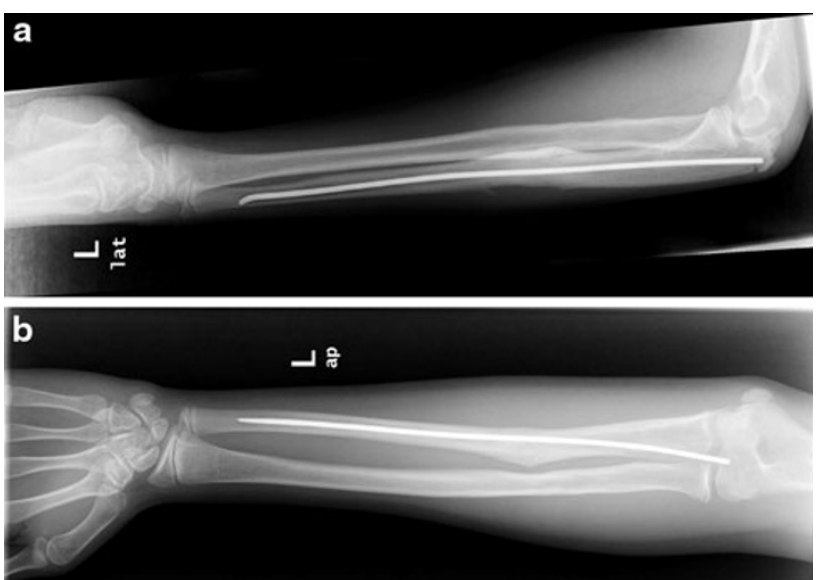

Fig. 3 a Complete union of ulna 4 months post ESIN-lateral view. b Complete union of ulna 4 months post ESIN—anteroposterior view

successfully treated diaphyseal forearm fractures in children using transphyseal intramedullary $\mathrm{K}$ wires [6]. They reported zero incidence of nonunion.

In our patient, the $\mathrm{K}$ wires were removed at 6 weeks followed by another 2 weeks of above elbow casting. We postulate that the vascular supply to the fracture site had recovered by 6 weeks. However, the total duration of immobilization of 8 weeks was probably insufficient, leading to the hypertrophic nonunion.

The use of ESIN in treatment of acute diaphyseal forearm fractures in children has been well described in the literature with favourable outcomes [7, 8]. Moez et al. [9] recently reported three cases of ulna nonunion following ESIN of which all occurred at the mid-shaft. The reduction method was an open reduction in all their cases. Our case 

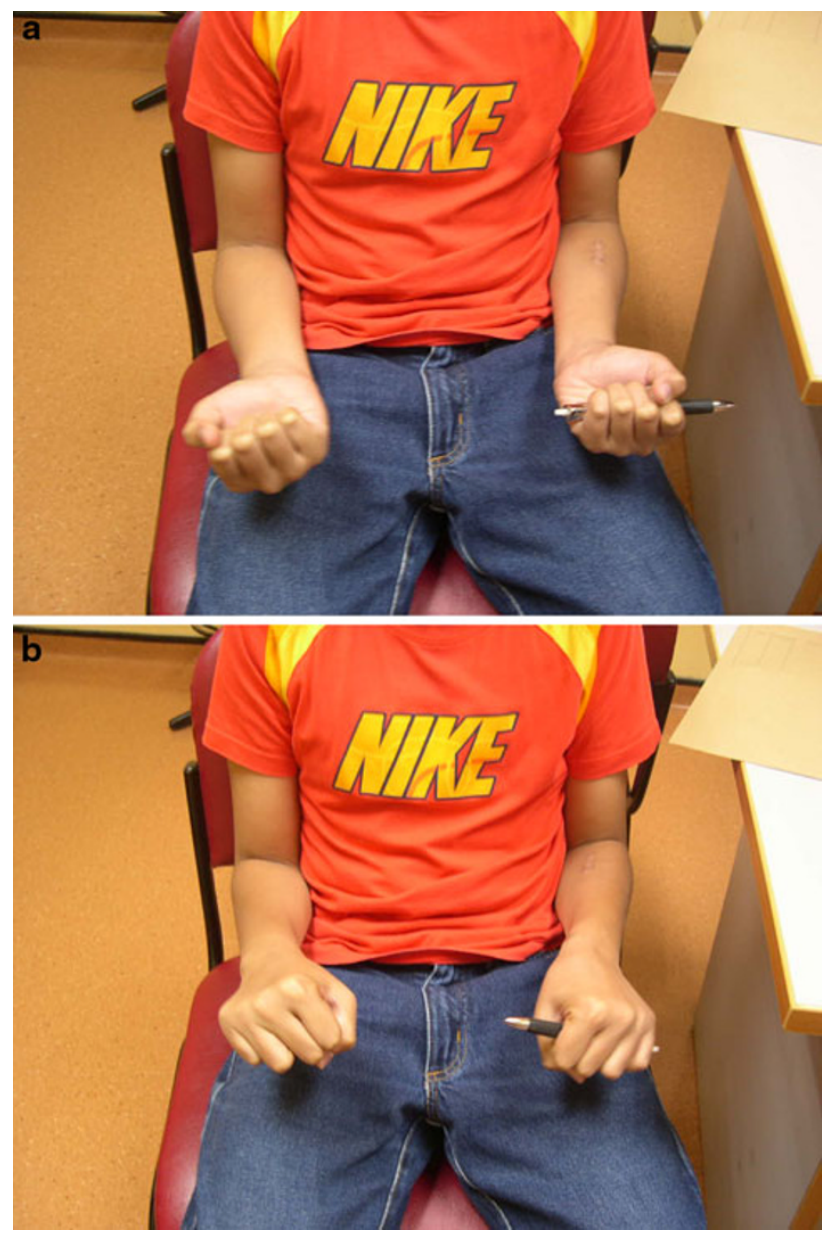

Fig. 4 a Left forearm in full supination. b Left forearm in full pronation

was similar as the fracture site was at the mid-shaft of ulna treated with open reduction.

Compression plating to treat nonunion has been advocated by several authors [2]. Two cases of nonunion reported by Moez et al. were both treated with plating following removal of ESIN [9]. However, we have not found any reports using ESIN to treat nonunion of diaphyseal forearm fractures in children as described in our case.

\section{Conclusion}

Open reduction of ulna shaft fracture has a risk of causing nonunion and is best avoided if possible. We believe that stable fixation with ESIN is adequate in treating hypertrophic nonunion in a child. We do not recommend open reduction and rigid fixation with a plate in children.

Open Access This article is distributed under the terms of the Creative Commons Attribution Noncommercial License which permits any noncommercial use, distribution, and reproduction in any medium, provided the original author(s) and source are credited.

\section{References}

1. Wilkins KE (2005) Principles of fracture remodeling in children. Injury 36(Suppl 1):A3-A11

2. Adamczyk MJ, Riley PM (2005) Delayed union and nonunion following closed treatment of diaphyseal pediatric forearm fractures. J Pediatr Orthop 25(1):51-55

3. Lewallen RP, Peterson HA (1985) Nonunion of long bone fractures in children: a review of 30 cases. J Pediatr Orthop 5(2):135-142

4. Wright TW, Glowczewskie F (1998) Vascular anatomy of the ulna. J Hand Surg [Am] 23(5):800-804

5. Giebel GD et al (1997) Arterial supply of forearm bones and its importance for the operative treatment of fractures. Surg Radiol Anat 19(3):149-153

6. Yung PS et al (2004) Percutaneous transphyseal intramedullary Kirschner wire pinning: a safe and effective procedure for treatment of displaced diaphyseal forearm fracture in children. $\mathbf{J}$ Pediatr Orthop 24(1):7-12

7. Garg NK et al (2008) Use of elastic stable intramedullary nailing for treating unstable forearm fractures in children. J Trauma 65(1):109-115

8. Jubel A et al (2005) Outcomes and complications of elastic stable intramedullary nailing for forearm fractures in children. J Pediatr Orthop B 14(5):375-380

9. Ballal MS et al (2009) Nonunion of the ulna after elastic stable intramedullary nailing for unstable forearm fractures: a case series. J Pediatr Orthop B 18(5):261-264 